\title{
Análisis psicométrico de la escala clima organizacional en servidores administrativos de una universidad pública ${ }^{1}$
}

\author{
José Manuel Calizaya-López² \\ Universidad Nacional de San Agustín de Arequipa \\ jcalizayal@unsa.edu.pe \\ Rildo Santos Bellido-Medina ${ }^{3}$ \\ Universidad Nacional de San Agustín de Arequipa \\ rbellidome@unsa.edu.pe \\ Julio Cesar Huamani-Cahua 4 \\ Universidad Nacional de San Agustín de Arequipa \\ jhuamanicah@unsa.edu.pe
}

DOI: https://doi.org/10.21158/01208160.n91.2021.3051

Fecha de recepción: 22 de febrero de 2021

Fecha de aprobación: 02 de agosto de 2021

Fecha de publicación: 30 de octubre de 2021

Cómo citar este artículo: Calizaya-López, J. M.; Bellido-Medina, R. S.; Huamani-Cahua, J. C. (2021). Análisis psicométrico de la escala clima organizacional en servidores administrativos de una universidad pública. Revista Escuela de Administración de Negocios, (91), páginas. DOI: https://doi.org/10.21158/01208160.n91.2021.3051

\section{Resumen}

El estudio dio origen a este trabajo tuvo como objetivo validar la escala clima organizacional en servidores administrativos de una universidad pública. El estudio es de tipo instrumental para valorar las propiedades psicométricas de la escala. Los participantes fueron 624 servidores administrativos de la universidad pública. Se elaboró el instrumento tomando como referencia otros instrumentos validados sobre el clima organizacional y se usaron indicadores de validez de contenido — cinco jueces expertos con la fórmula de la V de Aiken-. Además, se realizó el análisis factorial confirmatorio de primer y de segundo orden, y para el análisis de consistencia interna se utilizó el coeficiente de omega factorial. Los resultados muestran índices adecuados de validez de contenido para 75 ítems, mientras que el análisis factorial confirmatorio de primer orden mostró parámetros de ajuste apropiados luego de redefinir la escala para las tres dimensiones y los cincuenta ítems. El análisis de segundo orden no mostró adecuados indicadores de bondad de ajuste. Se analizaron las dimensiones como no correlacionadas — ortogonales-, de manera que se encontraron cargas factoriales altas en las tres dimensiones. Finalmente, la escala mostró una alta consistencia interna. Se concluye que la escala cuenta con una estructura factorial tridimensional e indicadores de validez y confiabilidad aceptables para la medición del clima organizacional.

Palabras clave: clima organizacional; medición de clima organizacional; cultura organizacional; diseño organizacional; gerencia de talento humano; talento humano en universidades.

${ }^{1}$ Proyecto financiado por la Universidad Nacional de San Agustín de Arequipa, Perú, según contrato n. 009-2016-UNAS.

2 Doctor en Ciencias de la Educación. Magíster en Gestión Social y Desarrollo Sostenible. Licenciado en Trabajo Social - Universidad Nacional de San Agustín de Arequipa. ORCID: https://orcid.org/0000-0001-6221-0909

${ }^{3}$ Doctor en Psicología. Magister en Gerencia estratégica de Recursos Humanos. Licenciado en Relaciones Industriales y Públicas - Universidad Nacional de San Agustín de Arequipa. ORCID: https://orcid.org/0000-0002-8699-3490

${ }^{4}$ Doctor en Ciencias Sociales. Magíster en Psicología -Clínica, Educativa, Infantil y adolescencia-- Psicólogo - Universidad Nacional de San Agustín de Arequipa. ORCID: https://orcid.org/0000-0001-8159-803X 


\title{
Psychometric Analysis of the Organizational Climate Scale in Administrative Employees at a Public University
}

\begin{abstract}
This study aimed to validate the organizational climate scale of administrative employees at a public university. It is an instrumental study to assess the scale's psychometric properties, featuring the participation of 624 administrative employees at the public university. The instrument was developed using other validated instruments on organizational climate as a reference and content validity indicators-five expert judges validated with Aiken's $\mathrm{V}$ formula. In addition, we performed first and second-order confirmatory factor analysis and used the omega factor coefficient to analyze internal consistency. The results show adequate content validity indices for 75 items. In comparison, the first-order confirmatory factor analysis showed appropriate fit parameters after redefining the scale for the three dimensions and the fifty items. The second-order analysis did not show adequate goodness-of-fit indicators. We analyzed the dimensions as uncorrelated-orthogonaland found high factor loadings in all three dimensions. Finally, the scale showed high internal consistency. We concluded that the scale has a three-dimensional factor structure and acceptable validity and reliability indicators to measure organizational climate.
\end{abstract}

Keywords: organizational climate; organizational climate measurement; organizational culture; organizational design; human talent management; human talent in universities.

Análise psicométrica da escala de clima organizacional em servidores administrativos de uma universidade pública

\section{Resumo}

0 estudo que deu origem a este trabalho teve como objetivo validar a escala de clima organizacional em servidores administrativos de uma universidade pública. 0 estudo é de caráter instrumental para avaliar as propriedades psicométricas da escala. Os participantes foram 624 servidores administrativos de uma universidade pública. 0 instrumento foi desenvolvido tomando como referência outros instrumentos validados sobre clima organizacional e indicadores de validade de conteúdo -cinco juízes especialistas com a fórmula $\mathrm{V}$ de Aiken-. Além disso, foi realizada a análise fatorial confirmatória de primeira e segunda ordem, e o coeficiente ômega fatorial foi utilizado para a análise de consistência interna. Os resultados mostram índices adequados de validade de conteúdo para 75 itens, enquanto a análise fatorial confirmatória de primeira ordem mostrou parâmetros de ajuste adequados após redefinir a escala para as três dimensões e os cinquenta itens. A análise de segunda ordem não mostrou indicadores adequados de qualidade de ajuste. As dimensões foram analisadas como não correlacionadas -ortogonais-, de modo que foram encontradas cargas fatoriais elevadas nas três dimensões. Finalmente, a escala apresentou alta consistência interna. Conclui-se que a escala possui estrutura fatorial tridimensional e indicadores de validade e confiabilidade aceitáveis para a medição do clima organizacional.

Palavras-chave: clima organizacional; medição do clima organizacional; cultura organizacional; desenho organizacional; gestão de talentos humanos; talento humano nas universidades. 
Analyse psychométrique du climat organisationnel des personnels administratifs d'une université publique

\begin{abstract}
Résumé
Cette étude vise à valider l'échelle du climat organisationnel des personnels administratifs d'une université publique. L'étude évalue les propriétés psychométriques de l'échelle à laquelle ont participé 624 fonctionnaires administratifs de l'université publique. L'instrument de mesure mis en place prend comme référence d'autres instruments validés pour le climat organisationnel ainsi que des indicateurs de validité de contenus - cinq juges experts de la formule $\mathrm{V}$ d'Aiken. Une analyse factorielle confirmatoire de premier et deuxième ordre a été réalisée et un coefficient factoriel oméga utilisé pour l'analyse de la cohérence interne. Les résultats montrent des indices de validité de contenu satisfaisants pour 75 items, tandis que l'analyse factorielle confirmatoire de premier ordre montre des paramètres d'ajustement appropriés après redéfinition de l'échelle pour trois dimensions et cinquante items. L'analyse de second ordre n'a pas montré d'indicateurs adéquats de qualité d'ajustement. Les trois dimensions ont été analysées comme non corrélées — orthogonales - de sorte que des saturations factorielles élevées y ont été obtenues. L'échelle a montré une cohérence interne élevée. En conclusion, cette échelle possède une structure factorielle tridimensionnelle et des indicateurs de validité et de fiabilité acceptables pour la mesure du climat organisationnel.
\end{abstract}

Mots-clés: climat organisationnel; mesure du climat organisationnel; culture organisationnelle; design organisationnel; gestion des ressources humaines; ressources humaines dans les universités.

\title{
1. Introducción
}

El clima organizacional es considerado el conjunto de percepciones globales que exterioriza el colaborador de su organización, tomando en consideración el comportamiento humano, la estructura y los procesos organizacionales que se producen en su medio laboral (GonzálesVásquez et al., 2021).

Sin embargo, la mayoría de los estudios relacionados con el tema se han desarrollado en el sector empresarial, de modo que existen pocos estudios en el sector educación, sobre todo del ámbito público académico (Botello-Ramirez, Beltrán-González y Cárdenas-Domínguez, 2021; Montoya-Cáceres et al., 2017). Además, la misma naturaleza de estas organizaciones educativas públicas mantiene una estructura cerrada que dificulta la explicación de cómo percibe el servidor este clima en el ámbito de trabajo que difiere según la edad, el sexo, el puesto y la condición de trabajo (Calizaya-López y Bellido-Medina, 2020). 
Según información sobre clima organizacional, este es clave en aquellas organizaciones que buscan incrementar sus sistemas de producción a través de la eficacia y la optimización del servicio brindado hacia el cliente. Así mismo, se evidencia que no existe un acuerdo en la definición y en sus dimensiones entre los investigadores, quienes se refieren, por un lado, a factores organizacionales de naturaleza objetiva, como, por ejemplo, la estructura, las políticas y las reglas, y por otro a aspectos de carácter subjetivo, tales como la cordialidad y el apoyo (Iglesias-Armenteros y Sánchez-García, 2015; Pecino-Medina et al., 2015; SoteloAsef y Figueroa-González, 2017).

Autores como Palafox-Soto, Ochoa-Jiménez y Jacobo-Hernández (2019) enfatizan en la presencia de un ambiente interpersonal de seguridad, sinceridad y calma en la organización ante situaciones de conflicto. Así mismo, el clima de la organización ha sido considerado una variable que origina efectos importantes sobre la motivación, la satisfacción y la productividad (Charry-Condor, 2018).

Para otros investigadores es una variable que se condiciona como la antigüedad en el trabajo, la edad, el género o las condiciones del trabajo, entre otras (Bernal-González, Pedraza-Melo y Sánchez-Limón, 2015; García-Velázquez et al., 2014; Meza-Barajas, 2017). Mientras que, según otros autores, se trata de una variable interviniente que se encarga de mediar entre los contextos sociales, la organización empresarial y la conducta individual del colaborador (Del Toro-Granados, Salazar-Sotter y Gómez-Rangel, 2011; González-Bonilla, 2019; GonzálezMillán, Rodríguez-Díaz y Molina-Valencia, 2011).

En la medición del clima organizacional se han evidenciado algunas dimensiones comunes tales como a) la autonomía de los colaboradores dentro del sistema, b) la estructura y los deberes de los miembros por su puesto de trabajo, c) las recompensas recibidas del empleador, y d) la valoración que recibe el colaborador de sus superiores (Riveros-Paredes y Grimaldo-Muchotrigo, 2017), de modo que se propone que estas dimensiones deben reflejar la imagen del clima organizacional. 
Bajo ese contexto se han desarrollado una serie de instrumentos de medición en el ámbito latinoamericano, sobre todo en Colombia (Castillo-Martín, 2013; Gómez, 2001; Gómez-Rada, 2004; Toro-Álvarez, 2001). En el contexto educativo se destaca el trabajo de AlcántarEnríquez, Maldonado-Radillo y Arcos-Vega (2012), quienes validaron una escala para determinar el ambiente de trabajo percibido por los empleados de una universidad pública. Tapias-Cuadrado (2014) diseñó un cuestionario que integra las dimensiones del clima organizacional expuestas por Chiang-Vega et al. (2008): autonomía, cohesión, confianza, presión, apoyo, reconocimiento, equidad e innovación.

En México, Hernández-Sampieri y Méndez-Valencia (2012) utilizaron el análisis factorial para evaluar el clima y la cultura organizacional centrado en el modelo de los valores en competencia, y Williams (2013) diseñó una escala sobre clima laboral con cinco dimensiones del comportamiento organizacional: liderazgo, comunicación, motivación, espacio físico y trabajo en equipo.

En el caso peruano no se encontraron instrumentos validados para la evaluación del clima organizacional en contextos públicos, solo se encontró una propuesta creada por el MINSA (Ministerio de Salud del Perú, 2011), organismo que desarrolló una escala que toma en cuenta los factores de potencial humano - liderazgo, innovación, recompensa y confort-, diseño organizacional -estructura, toma de decisiones, comunicación organizacional y remuneración-, y cultura organizacional -identidad, conflicto y cooperación y motivación-; sin embargo, no ha mostrado indicadores importantes psicométricos en su uso (Fernández-Oliva et al., 2019).

Por tanto, se planteó como objetivo validar la escala clima organizacional en servidores administrativos de una universidad pública, considerando aquellas dimensiones que se han mostrado pertinentes en el análisis del clima organizacional en otros contextos y los aportes de los trabajos desarrollados en Perú. De acuerdo con la estructura, a continuación se describen los apartados sobre la metodología utilizada, los principales resultados del análisis estadístico, la discusión de resultados, las conclusiones y las referencias bibliográficas. 


\section{Metodología}

El estudio es de tipo instrumental, con la intención de valorar las propiedades psicométricas de la escala clima organizacional, a fin de estar en capacidad de aplicarlo en el campo de la evaluación de este constructo en servidores administrativos de universidades públicas.

Los participantes fueron 624 servidores de una universidad pública $-57,9 \%$ varones y $42,1 \%$ mujeres - con una edad $-\mathrm{M}=46,50 ; \mathrm{DE}=11,91-$ dentro de un rango de 17 a 73 años. Con respecto a su estado civil, el 39,1 \% son solteros, el $54 \%$ son casados, el 2,2 \% son convivientes, el $0,5 \%$ son divorciados y el $1,4 \%$ son viudos; hubo un porcentaje que no evidenció su estado civil. Según escolaridad, el 0,5 \% estudios primarios, el 10,6 \% estudios secundarios, el 20,5 \% formación técnica de nivel superior, el $66 \%$ estudios universitarios y el 2,4\% no indicó sus estudios.

Según variables laborales, los servidores señalaron que el 59,9 \% tiene relación laboral estable con la institución -nombrados-, el 24 5\% tiene una modalidad de contrato CAS contrato administrativo de servicios-, el 9,8\% contrato temporal, el 0,6\% recibió por honorarios vía una actividad concreta y limitada en el tiempo, y el 5,1\% no evidencia su situación laboral. El método para seleccionar la muestra fue probabilístico, a través de la técnica de muestreo estratificado (Hernández, Fernández y Baptista, 2014).

El instrumento se construyó teniendo en cuenta los instrumentos elaborados por Williams (2013), Alcántar-Enríquez, Maldonado-Radillo y Arcos-Vega (2012), Hernández-Sampieri y Méndez-Valencia (2012), Tapias-Cuadrado (2014), y el Ministerio de Salud del Perú (MINSA, 2011). Inicialmente, el instrumento contaba con 112 ítems, organizados en tres dimensiones y dieciocho factores. La dimensión Clima organizacional estuvo conformada por nueve factores y sesenta ítems; la dimensión Diseño organizacional la conformaron tres factores y dieciocho ítems; la dimensión Potencial humano conformada por seis factores y 34 ítems (véase la Tabla 1). La forma de respuesta considera que 1 indica que la situación descrita no se asemeja en absoluto a la realidad de su organización, y 5 es el grado máximo de similitud (Likert, 1967). 
Una vez conformada la escala se sometió a un análisis de validez de contenido por cinco jueces expertos con la fórmula de la $\mathrm{V}$ de Aiken, a través del programa Visual Basic desarrollado por Merino-Soto y Livia-Segovia (2009). Luego de determinar su validez de contenido, se aplicó a los sujetos que conforman la muestra. Los datos fueron recogidos entre los servidores administrativos de la universidad pública de Arequipa.

Se aplicó el instrumento después de informar el objetivo del estudio y las condiciones de confidencialidad de sus datos, para lo cual autorizaron su participación a través del consentimiento informado. Para el análisis de validez se utilizó el análisis factorial confirmatorio (AFC), a través del Analysis of a Moment Structures (AMOS), versión 25. Con este propósito se empleó el método de máxima verosimilitud (ML) (Lara-Hormigo, 2014).

De manera preliminar se analizaron los ítems media, desviación estándar, curtosis, asimetría y correlación ítem-test, para, posteriormente, modelar la estructura factorial tomando como referencia la estructura de las dimensiones propuestas por los autores. Se verificó el modelo a través de las medidas de bondad de ajuste. Se ha propuesto utilizar el criterio $\chi 2 / \mathrm{df}<2$ ó $\chi 2 / \mathrm{df}<5$ como medida de ajuste (Schumacker y Lomax, 2004).

El índice de bondad de ajuste (GFI) y el índice de ajuste comparativo (CFI) en los que los valores superiores a 0,90 indican buen ajuste (Ruiz, Pardo y San Martin, 2010). El error cuadrático medio de aproximación (RMSEA), donde los valores de hasta 0,08 indican un ajuste razonable (Kline, 2011); en caso valores bajos de bondad de ajuste, se consideró eliminar aquellos ítems que no aportaban al modelo. Además, se ejecutó un análisis factorial confirmatorio (AFC) de segundo orden y la técnica de parceling (Marsh et al., 2013) en caso de que se obtenga una solución impropia. Finalmente, se estimó la fiabilidad por medio de la omega factorial - $\omega-($ Ventura y Caycho, 2017).

\section{Resultados}

Los valores de la V de Aiken mostraron puntuaciones superiores a 0,8 (Escurra-Mayaute, 1988), de modo que queda la escala en 75 ítems, los mismos que presentamos en la tabla 1. 
Tabla 1. Dimensiones y factores de la Escala del clima organizacional después de la validez de contenido

\begin{tabular}{|c|c|c|}
\hline Dimensión & Factor & Ítems \\
\hline \multirow{9}{*}{$\begin{array}{l}\text { Cultura } \\
\text { organizacional }\end{array}$} & Motivación & $1,2,3,4,5$ \\
\hline & Identidad & $6,7,8,9$ \\
\hline & Presión & $10,11,12,13$ \\
\hline & Apoyo & $14,15,16,17$ \\
\hline & Equidad & $18,19,20,21$ \\
\hline & Comunicación & $22,23,24,25$ \\
\hline & Trabajo en equipo & $26,27,28,29$ \\
\hline & Organización & $30,31,32,33$ \\
\hline & Condiciones de trabajo & $34,35,36,37,38$ \\
\hline \multirow{3}{*}{$\begin{array}{l}\text { Diseño } \\
\text { organizacional }\end{array}$} & Comunicación organizacional & $39,40,43,44,45,46,47$ \\
\hline & Reconocimiento & $41,42,48,49,50,51$ \\
\hline & Espacio físico & $52,53,54,55,56$ \\
\hline \multirow{5}{*}{$\begin{array}{l}\text { Potencial } \\
\text { humano }\end{array}$} & Innovación & $57,58,59,60$ \\
\hline & Liderazgo & $61,62,63,64$ \\
\hline & Recompensa & $65,66,67$ \\
\hline & Autonomía & $68,69,70$ \\
\hline & Cohesión & $71,72,73,74,75$ \\
\hline
\end{tabular}

Fuente. Elaboración propia.

\subsection{Análisis descriptivo}

Los estadísticos descriptivos muestran que los ítems no presentan valores elevados de asimetría y curtosis, considerando que los valores dentro del umbral $\pm 1,5$ indican variaciones leves de la normal (Revilla, Acosta y Reyna, 2013), excepto los ítems 5, 6, 7, 8, que corresponden al factor identidad. Al analizar las correlaciones ítem-test la mayoría de las correlaciones presentan coeficientes superiores a 0,2 (Kline, 2011); sin embargo, se eliminaron los ítems 5, 9, 13, 33 por presentar correlaciones bajas y asimetría alta en algunos casos. 
Tabla 2. Análisis de los ítems

\begin{tabular}{|c|c|c|c|c|c|}
\hline Ítems & $M$ & $\sigma$ & $\mathrm{g}_{1}$ & $\mathrm{~g}_{2}$ & $r-\mathrm{i}-\mathrm{t}-$ \\
\hline Ítem 1 & 3,38 & 1,231 & $-0,31$ & $-0,8$ & $0,703^{* *}$ \\
\hline Ítem2 & 3,53 & 1,285 & $-0,635$ & $-0,6$ & $0,743^{* *}$ \\
\hline Ítem 3 & 2,47 & 1,3 & 0,41 & $-0,973$ & $0,821^{* *}$ \\
\hline Ítem 4 & 2,39 & 1,378 & 0,552 & $-0,978$ & $0,802^{* *}$ \\
\hline Ítem 5 & 4,59 & 0,733 & $-2,296$ & 6,418 & $0,229^{* *}$ \\
\hline Ítem 6 & 4,68 & 0,665 & $-1,886$ & 10,673 & $0,864^{* *}$ \\
\hline Ítem 7 & 4,53 & 0,805 & $-1,909$ & 5,003 & $0,881^{* *}$ \\
\hline Ítem 8 & 4,46 & 0,904 & $-1,997$ & 4,06 & $0,816^{* *}$ \\
\hline Ítem 9 & 3,28 & 1,056 & $-0,311$ & $-0,221$ & $0,093^{*}$ \\
\hline Ítem 10 & 2,68 & 1,246 & 0,195 & $-0,949$ & $0,767^{* *}$ \\
\hline Ítem 11 & 2,5 & 1,285 & 0,458 & $-0,813$ & $0,750^{* *}$ \\
\hline Ítem 12 & 2,59 & 1,284 & 0,3 & $-0,937$ & $0,421^{* *}$ \\
\hline Ítem 13 & 3,15 & 1,37 & $-0,175$ & $-1,156$ & 0.036 \\
\hline Ítem 14 & 2,58 & 1,243 & 0,309 & $-0,865$ & $0,729^{* *}$ \\
\hline Ítem 15 & 3,18 & 1,251 & $-0,211$ & $-0,901$ & $0,852^{* *}$ \\
\hline Ítem 16 & 4,04 & 1,178 & $-1,216$ & 0,587 & $0,721^{* *}$ \\
\hline Ítem 17 & 3,74 & 1,227 & $-0,789$ & $-0,299$ & $0,734^{* *}$ \\
\hline Ítem 18 & 2,7 & 1,297 & 0,179 & $-1,037$ & $0,634^{* *}$ \\
\hline Ítem 19 & 3,24 & 1,414 & $-0,301$ & $-1,171$ & $0,731^{* *}$ \\
\hline Ítem 20 & 3,54 & 1,238 & $-0,513$ & $-0,635$ & $0,767^{* *}$ \\
\hline Ítem 21 & 3,47 & 1,277 & $-0,507$ & $-0,733$ & $0,651^{* *}$ \\
\hline Ítem 22 & 3,55 & 1,203 & $-0,526$ & $-0,589$ & $0,746^{* *}$ \\
\hline Ítem 23 & 3,28 & 1,294 & $-0,295$ & $-0,942$ & $0,656^{* *}$ \\
\hline Ítem 24 & 3,1 & 1,243 & $-0,032$ & $-0,948$ & $0,692^{* *}$ \\
\hline Ítem 25 & 3,66 & 1,153 & $-0,634$ & $-0,357$ & $0,487^{* *}$ \\
\hline Ítem 26 & 3,37 & 1,204 & $-0,308$ & $-0,746$ & $0,867^{* *}$ \\
\hline Ítem 27 & 3,44 & 1,194 & $-0,351$ & $-0,748$ & $0,881^{* *}$ \\
\hline Ítem 28 & 3,68 & 1,157 & $-0,688$ & $-0,292$ & $0,871^{* *}$ \\
\hline Ítem 29 & 3,65 & 1,134 & $-0,58$ & $-0,386$ & $0,643^{* *}$ \\
\hline Ítem 30 & 3,49 & 1,154 & $-0,449$ & $-0,576$ & $0,788^{* *}$ \\
\hline Ítem 31 & 4 & 1,064 & $-1,006$ & 0,42 & $0,764^{* *}$ \\
\hline Ítem 32 & 3,35 & 1,341 & $-0,351$ & $-0,991$ & $0,710^{* *}$ \\
\hline Ítem 33 & 2,14 & 1,357 & 0,833 & $-0,65$ & $0,098^{*}$ \\
\hline Ítem 34 & 3,38 & 1,251 & $-0,344$ & $-0,825$ & $0,735^{* *}$ \\
\hline Ítem 35 & 2,61 & 1,306 & 0,32 & $-1,01$ & $0,712^{* *}$ \\
\hline Ítem 36 & 2,93 & 1,209 & $-0,073$ & $-0,875$ & $0,791^{* *}$ \\
\hline Ítem 37 & 3,1 & 1,293 & $-0,141$ & $-1,05$ & $0,692^{* *}$ \\
\hline Ítem 38 & 3,49 & 1,267 & $-0,522$ & $-0,707$ & $0,311^{* *}$ \\
\hline Ítem 39 & 3,03 & 1,297 & $-0,111$ & $-1,051$ & $0,656^{* *}$ \\
\hline Ítem 40 & 2,73 & 1,22 & 0,105 & $-0,942$ & $0,531^{* *}$ \\
\hline Ítem 41 & 2,44 & 1,244 & 0,345 & $-1,007$ & $0,537^{* *}$ \\
\hline Ítem 42 & 3,78 & 1,155 & $-0,848$ & 0,057 & $0,622^{* *}$ \\
\hline Ítem 43 & 4,1 & 0,994 & $-1,144$ & 0,964 & $0,566^{* *}$ \\
\hline Ítem 44 & 3,31 & 1,33 & $-0,296$ & $-1,013$ & $0,794^{* *}$ \\
\hline Ítem 45 & 3,39 & 1,328 & $-0,375$ & $-1,004$ & $0,791^{* *}$ \\
\hline Ítem 46 & 4,21 & 0,949 & $-1,433$ & 2,129 & $0,607^{* *}$ \\
\hline Ítem 47 & 3,31 & 1,397 & $-0,329$ & $-1,128$ & $0,772^{* *}$ \\
\hline Ítem 48 & 2,8 & 1,399 & 0,159 & $-1,221$ & $0,426^{* *}$ \\
\hline
\end{tabular}




\begin{tabular}{|c|c|c|c|c|c|}
\hline Ítem 49 & 2,71 & 1,292 & 0,172 & $-1,037$ & $0,691^{* *}$ \\
\hline Ítem 50 & 3,22 & 1,181 & $-0,206$ & $-0,698$ & $0,556^{* *}$ \\
\hline Ítem 51 & 3,38 & 1,333 & $-0,411$ & $-1,005$ & $0,608^{* *}$ \\
\hline Ítem 52 & 3,02 & 1,374 & $-0,143$ & $-1,2$ & $0,691^{* *}$ \\
\hline Ítem 53 & 3,18 & 1,268 & $-0,274$ & $-0,897$ & $0,708^{* *}$ \\
\hline Ítem 54 & 2,65 & 1,34 & 0,182 & $-1,201$ & $0,676^{* *}$ \\
\hline Ítem 55 & 2,75 & 1,333 & 0,15 & $-1,126$ & $0,373^{* *}$ \\
\hline Ítem 56 & 3,5 & 1,214 & $-0,437$ & $-0,681$ & $0,538^{* *}$ \\
\hline Ítem 57 & 3,46 & 1,207 & $-0,415$ & $-0,653$ & $0,728^{* *}$ \\
\hline Ítem 58 & 3,11 & 1,262 & $-0,063$ & $-0,906$ & $0,745^{* *}$ \\
\hline Ítem 59 & 3,01 & 1,196 & $-0,038$ & $-0,805$ & $0,790^{* *}$ \\
\hline Ítem 60 & 3,34 & 1,195 & $-0,273$ & $-0,762$ & $0,752^{* *}$ \\
\hline Ítem 61 & 3,23 & 1,273 & $-0,308$ & $-0,95$ & $0,784^{* *}$ \\
\hline Ítem 62 & 3,39 & 1,266 & $-0,382$ & $-0,838$ & $0,751^{* *}$ \\
\hline Ítem 63 & 2,48 & 1,216 & 0,355 & $-0,838$ & $0,387^{* *}$ \\
\hline Ítem 64 & 1,96 & 1,181 & 1,002 & $-0,105$ & $0,575^{* *}$ \\
\hline Ítem 65 & 2,95 & 1,248 & $-0,112$ & $-0,935$ & $0,782^{* *}$ \\
\hline Ítem 66 & 2,05 & 1,191 & 0,861 & $-0,281$ & $0,751^{* *}$ \\
\hline Ítem 67 & 3,19 & 1,262 & $-0,273$ & $-0,835$ & $0,707^{* *}$ \\
\hline Ítem 68 & 3,09 & 1,264 & $-0,206$ & $-0,928$ & $0,759^{* *}$ \\
\hline Ítem 69 & 3,64 & 1,202 & $-0,748$ & $-0,204$ & $0,756^{* *}$ \\
\hline Ítem 70 & 4,21 & 0,871 & $-1,218$ & 1,682 & $0,603^{* *}$ \\
\hline Ítem 71 & 4,27 & 0,889 & $-1,473$ & 2,496 & $0,546^{* *}$ \\
\hline Ítem 72 & 3,5 & 1,15 & $-0,452$ & $-0,47$ & $0,680^{* *}$ \\
\hline Ítem 73 & 3,61 & 1,195 & $-0,573$ & $-0,526$ & $0,813^{* *}$ \\
\hline Ítem 74 & 3,26 & 1,251 & $-0,283$ & $-0,849$ & $0,821^{* *}$ \\
\hline Ítem 75 & 3,47 & 1,231 & $-0,471$ & $-0,656$ & $0,797^{* *}$ \\
\hline
\end{tabular}

Nota: $n=413 ; M=$ media; $\sigma=$ varianza; $g_{1}=$ asimetría; $g_{2}=$ curtosis; $r-i-t$ - = correlación ítem-test.

** $p<.01$-bilateral一.

Fuente. Elaboración propia.

\subsection{Análisis factorial confirmatorio}

El análisis factorial confirmatorio (AFC) se realizó para confirmar las dimensiones de cultura organizacional, diseño organizacional y potencial humano. Se analizó el modelo de nueve factores de la dimensión Cultura Organizacional (véase la Tabla 3), la cual no mostró un buen ajuste. Por este motivo se reespecificó el modelo, encontrando baja saturación y ambigüedad en los ítems de los factores presión -ítems 10, 11, 12 y 13- y el factor comunicación ítems 22, 23, 24, 25-, razón por la cual esos factores fueron eliminados.

Además, se eliminó el ítem 9 del factor Identidad, el ítem 14 del factor apoyo, el ítem 29 del factor trabajo en equipo, el ítem 33 del factor organización y el ítem 38 del factor condiciones de trabajo, es decir, aquellos que presentaban valores residuales mayores a $\pm 2,58$ (Hair, 
Anderson, Tatham y Black, 2014). Eliminados dos factores y trece ítems, se analizó el modelo de siete factores y veinticuatro ítems, encontrando un ajuste apropiado, $\chi 2=653,513 ; \mathrm{gl}=$ 230; $\mathrm{CFI}=0,939 ; \mathrm{GFI}=0,915 ; \mathrm{RMSEA}=0,054$ [IC90 \% 0,049, 0,059].

En la figura 1 se muestran las cargas factoriales estandarizadas de la dimensión Cultura organizacional, la misma que presenta una estructura multidimensional de siete factores, de modo que se encuentran cargas factoriales $-\lambda \geq 0,5-$ como adecuados (Johnson y Stevens, 2001); además, el promedio de las cargas factoriales en los factores Identidad - 0,80-, Apoyo -0,79- y Trabajo en equipo - 0,84- son mayores a 0,7 (Hair et al., 2014); en los demás factores como Motivación -0,61-, Equidad -0,61-, Organización -0,64- y Condiciones de trabajo - 0,67- los valores promedios de las cargas factoriales son próximas a 0,7, así como las correlaciones entre las variables no muestran multicolinealidad.

Figura 1. Análisis factorial confirmatorio de la dimensión Cultura organizacional

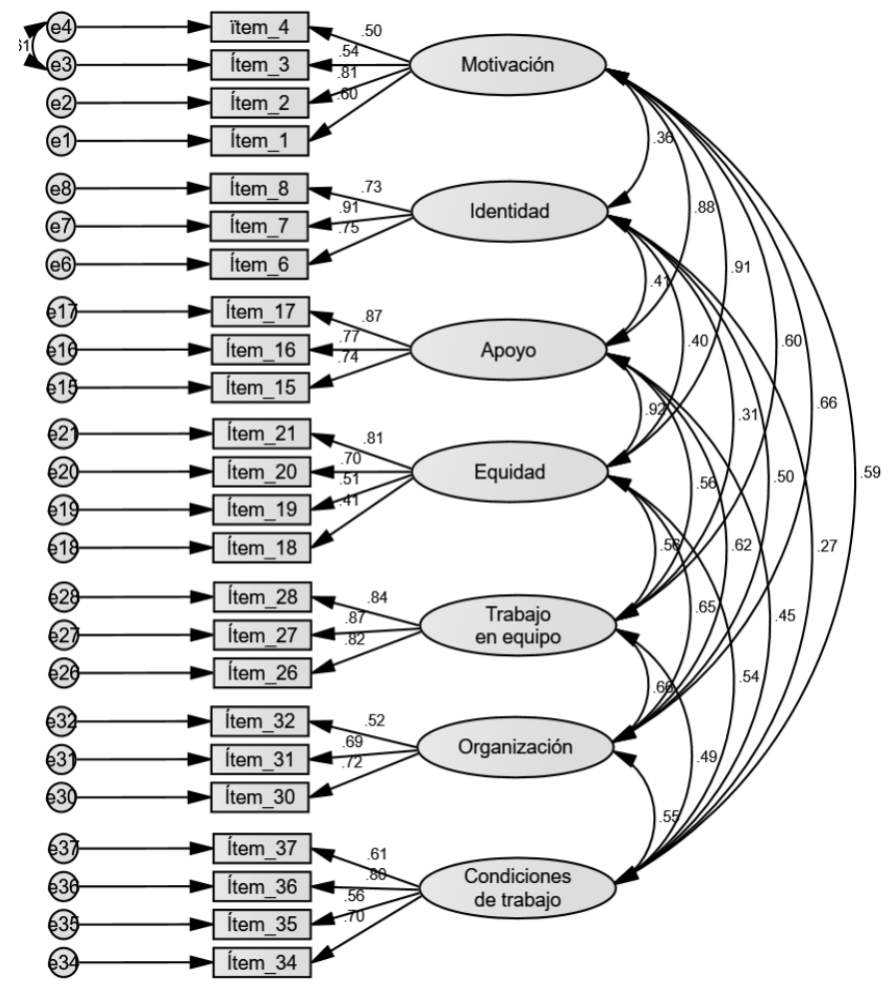

Fuente. Elaboración propia. 
En la tabla 3 se analizó el modelo de tres factores y dieciocho ítems de la dimensión Diseño organizacional, la misma que no muestra un buen ajuste. Por este motivo se reespecificó el modelo, encontrando baja saturación y valores residuales mayores a $\pm 2,58$ en los ítems 40 , 43 y 44 del factor Comunicación organizacional, los ítems 48 y 51 del factor Reconocimiento y el ítem 55 del factor Espacio físico, los cuales fueron eliminados. Se volvió a analizar el modelo y se encontró un ajuste apropiado, $\chi 2=225,512$; $\mathrm{gl}=50$; $\mathrm{CFI}=0,907$; GFI $=0,944$; RMSEA = 0,075 [IC90\% 0,065, 0,085].

Tabla 3. Cargas factoriales de la solución estandarizada del análisis factorial confirmatorio para el modelo final

\begin{tabular}{|c|c|c|}
\hline & Cultura organizacional & $\lambda$ \\
\hline & Motivación & \\
\hline 1 & Mi centro de labores me ofrece la oportunidad de hacer lo que mejor sé hacer. & 0,60 \\
\hline 2 & Mi jefe inmediato se preocupa por crear un ambiente laboral agradable. & 0,81 \\
\hline 3 & Me brindan reconocimiento por un buen desempeño laboral. & 0,54 \\
\hline \multirow[t]{2}{*}{4} & Reconocen mi esfuerzo si trabajo más de las horas reglamentarias. & 0,50 \\
\hline & Identidad & \\
\hline 6 & Me interesa el desarrollo de la Universidad. & 0,75 \\
\hline 7 & Me siento contento de ser parte del desarrollo de la Universidad. & 0,91 \\
\hline \multirow[t]{2}{*}{8} & Estoy satisfecho y orgulloso de trabajar en la Universidad. & 0,73 \\
\hline & Apoyo & \\
\hline 15 & En la Universidad los jefes cuidan a su personal. & 0,74 \\
\hline 16 & Mi jefe me respalda y deja que aprenda de mis errores. & 0,77 \\
\hline \multirow[t]{2}{*}{17} & Mi jefe me trata con respeto. & 0,87 \\
\hline & Equidad & \\
\hline 18 & Puedo contar con un trato justo por parte de mi jefe. & 0,41 \\
\hline 19 & Los directivos tratan igual a todos los que trabajan en la Universidad. & 0,51 \\
\hline 20 & Mi jefe no tiene favoritismos. & 0,70 \\
\hline \multirow[t]{2}{*}{21} & Si mi jefe sanciona a alguien es porque probablemente esa persona se lo merece. & 0,81 \\
\hline & Trabajo en equipo & \\
\hline 26 & Los objetivos están claramente establecidos en mi equipo de trabajo. & 0,82 \\
\hline 27 & El apoyo y escucha son frecuentes en mi equipo de trabajo. & 0,87 \\
\hline \multirow[t]{2}{*}{28} & Se hace presente el intercambio de ideas en mi equipo de trabajo. & 0,84 \\
\hline & Organización & \\
\hline 30 & Las labores que se desarrollan en mi área de trabajo se encuentran organizadas. & 0,72 \\
\hline 31 & $\begin{array}{l}\text { La documentación — procedimientos, instructivos y formatos- son útiles y apropiadas para } \\
\text { realizar mi trabajo. }\end{array}$ & 0,69 \\
\hline \multirow[t]{2}{*}{32} & $\begin{array}{l}\text { Conozco como se encuentra estructurada mi área de trabajo y cuáles son las actividades que } \\
\text { se desarrollan. }\end{array}$ & 0,52 \\
\hline & Condiciones de trabajo & \\
\hline 34 & En mi centro de trabajo existen accesos adecuados para el personal discapacitado. & 0,70 \\
\hline 35 & $\begin{array}{l}\text { Las áreas de trabajo se conservan limpias y en orden, permitiendo el desarrollo de mis } \\
\text { actividades. }\end{array}$ & 0,56 \\
\hline 36 & Dispongo de suficientes instalaciones sanitarias y áreas de descanso. & 0,80 \\
\hline 37 & En general, las condiciones de trabajo de mi área son seguras. & 0,61 \\
\hline
\end{tabular}




\begin{tabular}{|c|c|c|}
\hline & Diseño organizacional & $\lambda$ \\
\hline & Comunicación organizacional & \\
\hline 39 & Mi jefe inmediato trata de obtener información antes de tomar una decisión. & 0,54 \\
\hline 45 & $\begin{array}{l}\text { Mi jefe inmediato se comunica regularmente con los trabajadores para recabar apreciaciones } \\
\text { técnicas o percepciones relacionadas con el trabajo. }\end{array}$ & 0,76 \\
\hline 46 & Mi jefe inmediato me comunica si estoy realizando mi trabajo, bien o mal. & 0,52 \\
\hline \multirow[t]{2}{*}{47} & Presto atención a los comunicados que emiten mis jefes. & 0,78 \\
\hline & Reconocimiento & \\
\hline 41 & Mi salario y beneficios son razonables. & 0,31 \\
\hline 42 & Mi remuneración es adecuada en relación con el trabajo que realizo. & 0,54 \\
\hline 49 & Se ofrecen posibilidades para el desarrollo personal de los trabajadores. & 0,53 \\
\hline \multirow[t]{2}{*}{50} & Mi jefe se fija más en las habilidades de los trabajadores que en sus debilidades. & 0,54 \\
\hline & Espacio físico & \\
\hline 52 & La iluminación en mi espacio de trabajo es: & 0,61 \\
\hline 53 & El servicio de aseo en mi espacio de trabajo es: & 0,72 \\
\hline 54 & El equipo para realizar mi trabajo es: & 0,53 \\
\hline \multirow[t]{3}{*}{56} & El ruido para el desarrollo de mis actividades es: & 0,42 \\
\hline & Potencial humano & $\lambda$ \\
\hline & Innovación & \\
\hline 57 & La innovación es característica de la Universidad. & 0,62 \\
\hline 58 & Mis compañeros de trabajo toman iniciativas para la solución de problemas. & 0,56 \\
\hline 59 & En la Universidad todos están abiertos al cambio. & 0,71 \\
\hline \multirow[t]{2}{*}{60} & En la Universidad continuamente se adoptan nuevos y mejores métodos para hacer el trabajo. & 0,71 \\
\hline & Liderazgo & \\
\hline 61 & Existe una clara indicación de las funciones que cada uno debe desempeñar. & 0,84 \\
\hline 62 & Mi jefe inmediato me permite participar en la planificación del trabajo. & 0,82 \\
\hline \multirow[t]{2}{*}{64} & A menudo se inician trabajos que no se sabe porque se hacen. & 0,30 \\
\hline & Autonomía & \\
\hline 68 & Tengo autonomía laboral. & 0,59 \\
\hline 69 & Tengo libertad en la toma de decisiones. & 0,41 \\
\hline \multirow[t]{2}{*}{70} & Organizo mi trabajo como mejor me parece. & 0,49 \\
\hline & Cohesión & \\
\hline 72 & Promuevo un ambiente amigable dentro de la Universidad. & 0,51 \\
\hline 73 & Existe amistad y cariño entre los miembros de la Universidad. & 0,77 \\
\hline 74 & Tengo libertad para comunicarme abiertamente con mis superiores. & 0,81 \\
\hline 75 & Existe buena relación entre los superiores y subordinados. & 0,78 \\
\hline
\end{tabular}

Fuente. Elaboración propia.

En la figura 2 se muestran las cargas factoriales estandarizadas de la dimensión Diseño organizacional, con una estructura multidimensional de tres factores, encontrando cargas factoriales $-\lambda \geq 0,5-$, excepto en los ítems 41 del factor Reconocimiento e ítem 56 del factor Espacio físico — sin embargo, son cargas factoriales aceptables—; además, el promedio de las cargas factoriales en los factores Comunicación organizacional - 0,65-, Reconocimiento 0,48- y Espacio físico - 0,57- son mayores a 0,7. 
Figura 2. Análisis factorial confirmatorio de la dimensión Diseño organizacional

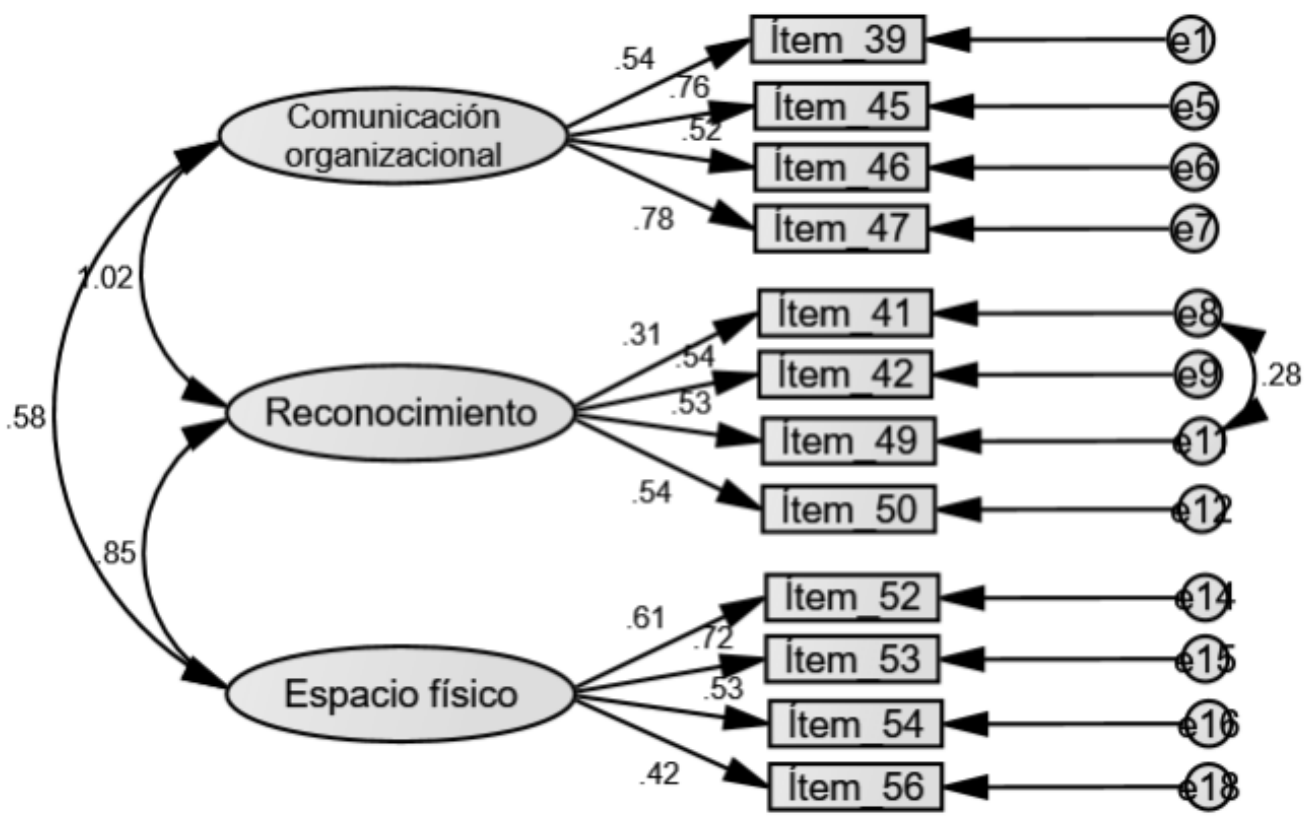

Fuente. Elaboración propia.

Posteriormente, se analizó el modelo de cinco factores y veinte ítems de la dimensión Potencial humano, la cual no mostró un buen ajuste. Por este motivo se reespecificó el modelo, encontrando baja saturación y ambigüedad en los ítems del factor Recompensa eliminado-. Asimismo, los ítems con valores residuales mayores a $\pm 2,58$ son el 63 del factor Liderazgo y el ítem 71 del factor Cohesión —eliminados-. Se realizó el análisis de un nuevo modelo sin el factor e ítems eliminados, encontrando buenos ajustes, $\chi 2=320,273$; gl $=71$; $\mathrm{CFI}=0,913 ; \mathrm{GFI}=0,928 ; \mathrm{RMSEA}=, 075$ [IC90\% 0,067, 0,084].

En la figura 3 se muestran las cargas factoriales estandarizadas de la dimensión potencial humano, con una estructura multidimensional de cuatro factores, encontrando cargas factoriales $-\lambda \geq 0,5-$, excepto en los ítems 64 del factor Liderazgo y los ítems 69 y 70 del factor Autonomía - sin embargo, son cargas factoriales aceptables-; además, el promedio de las cargas factoriales en los factores Innovación -0,65-, Liderazgo - 0,65-, Cohesión - 0,70 - y Espacio físico - 0,57 - son próximas a 0,7 —adecuadas-, excepto en el factor 
Autonomía -0,50-, así mismo, las correlaciones entre las variables no muestran multicolinealidad.

Figura 3. Análisis factorial confirmatorio de la dimensión Potencial humano

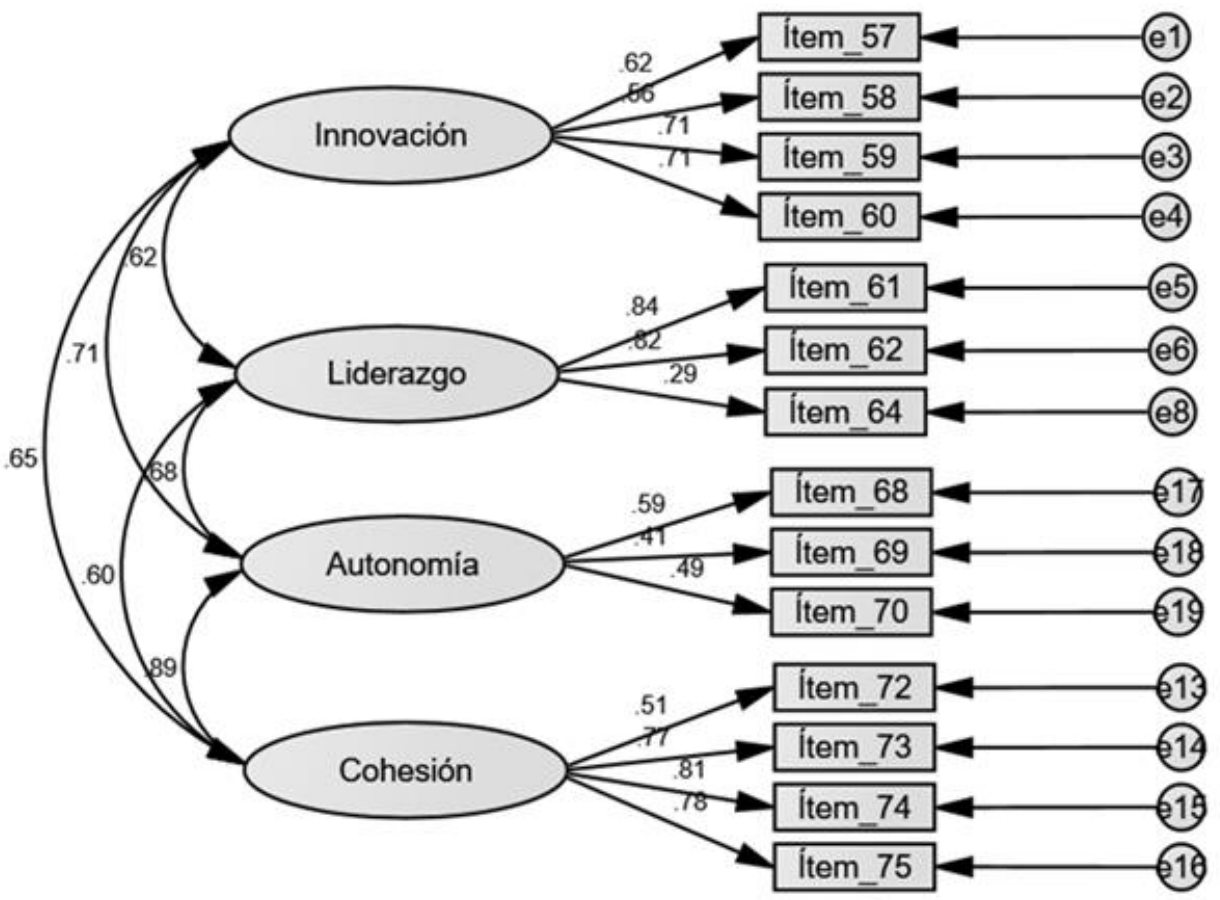

Fuente. Elaboración propia.

\subsection{Análisis factorial confirmatorio de segundo orden}

Para este análisis se consideraron los siete factores latentes de la dimensión Cultura organizacional, los cuatro factores latentes de la dimensión Diseño organizacional y los cuatro factores latentes de la dimensión Potencial humano, hallando un modelo que no presenta un buen ajuste, $\chi 2=4334,347$; $\mathrm{gl}=1158$; $\mathrm{CFI}=0,789 ; \mathrm{GFI}=0,779 ; \mathrm{RMSEA}=0,066$ [IC90 \% 0,064, ,068]. No se reespecificó el modelo. Las cargas factoriales del análisis factorial confirmatorio evidencian una solución impropia por presencia de casos Heywood —Diseño organizacional y Potencial humano-. 
Para precisar la solución impropia se aplicó la técnica de parceling (Marsh et al., 2013), que consiste en agrupar los ítems de cada factor, lo cual daría lugar a una variable observable que represente y explique cada dimensión. Así, se encuentra que existían correlaciones mayores a 1, fuera del rango, por tanto, impropias y mal definidas -Diseño organizacional y Potencial humano-; de igual modo, existen covarianzas entre el factor apoyo - Cultura organizacional-y el factor comunicación organizacional — factor Diseño organizacional-.

Además, del factor Condiciones de trabajo - Cultura Organizacional-y del factor Espacio físico —Diseño organizacional—, en ambas covarianzas pareciera que los ítems y factores miden la misma variable latente. A pesar de que el modelo presenta un buen ajuste, $\chi 2=$ 365,857; $\mathrm{gl}=$ 69; $\mathrm{CFI}=0,937 ; \mathrm{GFI}=0,920 ; \mathrm{RMSEA}=0,080$ [IC90\% 0,075, 0,092], debido a la presencia de valores impropios - casos Heywood- los resultados no pueden ser interpretados.

Finalmente, el análisis de los ítems, los factores y las dimensiones medidas se realizó por separado, siguiendo una rotación ortogonal que indica cómo las dimensiones no están correlacionadas. En la tabla 3 se muestran las cargas factoriales de las dimensiones y sus factores, analizadas de forma separada - Figura 1, 2 y 3-, las cuales muestran $\lambda$ entre 0,41 y 0,91 - excepto el ítem 64-; tampoco se presentan correlaciones entre las dimensiones.

\subsection{Análisis de confiabilidad}

Finalmente, los resultados de la confiabilidad por consistencia interna se estimaron por medio del omega - $\omega-$; se encontró que para la dimensión Cultura organizacional los factores presentan buenos índices de confiabilidad: Motivación $-\omega=0,71-$, Identidad $-\omega$ $=0,84-$, Apoyo $-\omega=0,837-$, Equidad $-\omega=0,71-$, Trabajo en equipo $-\omega=0,88-$, Organización $-\omega=0,68-$, y Condiciones de trabajo $-\omega=0,77-$. Con respecto a la dimensión Diseño organizacional, se presentan índices de confiabilidad aceptables superiores a 0,65 (Katz, 2006) en los factores Comunicación organizacional $-\omega=0,75-$, Reconocimiento $-\omega=0,55-$ y el factor Espacio físico $-\omega=0,66-$ Finalmente, en la dimensión Potencial humano los índices de confiabilidad son buenos en los factores 
Innovación $-\omega=0,75-$, Liderazgo $-\omega=0,72-$ y Cohesión $-\omega=0,80-$, sin embargo, el factor Autonomía presenta índices de confiabilidad bajos $-\omega=0,50-$, por lo cual la precisión e interpretación de este factor debería tomarse con mucha cautela.

\section{Discusión}

Se planteó como objetivo validar la escala clima organizacional en servidores administrativos para servidores públicos en Perú. Los resultados del análisis factorial confirmatorio (AFC) proporcionan apoyo empírico para el modelo tridimensional de la Escala del clima organizacional. Así mismo, las cargas factoriales de cada ítem son todas significativas, pues la mayoría de los ítems superan el valor de 0,70, que representan más de la mitad de la varianza explicada en cada ítem.

Este resultado significa que la varianza del error para cada ítem es menor que la varianza explicada por la escala del clima organizacional, lo que proporciona apoyo a la validez de constructo del instrumento (Hultell y Gustavsson, 2008). La diferencia entre las cargas factoriales de los ítems cuyas cargas factoriales son menores a 0,70 y aquellos mayores a este valor evidencian que los ítems influyen de forma diferente en la definición del constructo clima organizacional.

En ese sentido, en el factor equidad de la dimensión cultura organizacional los ítems 18 y 19 son los que menores cargas factoriales presentan, datos que indican cómo en este factor podría existir mayor error en las respuestas de los sujetos investigados. Lo mismo sucede en el ítem 32 del factor Organización «Conozco como se encuentra estructurada mi área de trabajo y cuáles son las actividades que se desarrollan», y en el ítem 35 del factor condiciones de trabajo «Las áreas de trabajo se conservan limpias y en orden, permitiendo el desarrollo de mis actividades». Son ítems con cargas factoriales bajas que indicarían mayor error.

Sin embargo, los ítems que explican mayor varianza en cada uno de los factores son el ítem dos del factor Motivación «Las áreas de trabajo se conservan limpias y en orden, permitiendo el desarrollo de mis actividades». En el factor identidad, el ítem 7, «Me siento contento de ser parte del desarrollo de la Universidad». En el factor Apoyo el ítem 16 «Mi jefe me respalda y 
deja que aprenda de mis errores». En el factor Equidad, el ítem 21 «Si mi jefe sanciona a alguien es porque probablemente esa persona se lo merece». En el factor Trabajo en Equipo, el ítem 27 «El apoyo y escucha son frecuentes en mi equipo de trabajo». En el factor Organización el ítem 30 «Las labores que se desarrollan en mi área de trabajo se encuentran organizadas» y en el factor Condiciones de trabajo el ítem 36 «Dispongo de suficientes instalaciones sanitarias y áreas de descanso» son los que mayor carga factorial presentan, de modo que pueden considerarse como ítems que representan la cultura organizacional de la institución investigada.

Si bien se presentaron algunas limitaciones como, por ejemplo, la colaboración de los servidores administrativos para ser encuestados, estas se superaron con la respectiva autorización de la Universidad, entendiendo que el estudio permitiría conocer claramente la percepción que tiene el servidor sobre el clima organizacional en la institución educativa.

\section{Conclusiones}

La percepción del servidor sobre la medición del clima organizacional expresa la apreciación que tiene de la Universidad, sobre todo, con respecto a la imagen, los valores, los procesos, la estructura y su recurso humano, indicadores que permiten conocer el tipo de ambiente laboral en el que se desempeña el servidor con la finalidad de implementar políticas institucionales que ayuden a mejorar las condiciones laborales y construir una organización pública de prestigio.

Se considera que esta primera aproximación psicométrica en el contexto peruano en el sector público permite un acercamiento a la medición de un constructo multidimensional sobre clima organizacional, el cual pone en evidencia las características y los lineamientos de la organización con las de la persona, muchas veces en conflicto, pero también en concordancia; todo esto con el fin de establecer un equilibrio que permita entender cómo la proporción entre los intereses de la organización y los personales deben mantenerse, de modo que se privilegie a la persona en la mayoría de sus decisiones. 
Si bien existen innumerables instrumentos del clima organizacional, no se cuenta con una medida valida y confiable de este en instituciones públicas académicas y en la muestra seleccionada para determinar con eficiencia el nivel de percepción del clima en este tipo de instituciones. En este sentido, el presente estudio sobre la validez de la escala pretende ser un aporte al sector académico.

Se puede concluir que la escala clima organizacional muestra indicadores aceptables de validez y confiabilidad para su uso en contextos administrativos públicos en Perú. Muestra una estructura factorial tridimensional y una alta consistencia interna en las dimensiones evaluadas. Recomendamos ampliar la muestra a otros contextos públicos y a servidores o colaboradores de organizaciones privadas para obtener mayores indicadores de validez del instrumento desarrollado.

\section{Referencias}

Alcántar-Enríquez, V.; Maldonado-Radillo, S.; Arcos-Vega, J. (2012). Medición del clima laboral requerido para asegurar la efectividad del sistema de gestión de calidad. Revista Internacional Administración y Finanzas, 5(3), 55- 68.

Bernal-González, I.; Pedraza-Melo, N.; Sánchez-Limón, M. (2015). El clima organizacional y su relación con la calidad de los servicios públicos de salud: diseño de un modelo teórico. Estudios Gerenciales, 31(134), 8-19. DOI: https://doi.org/10.1016/j.estger.2014.08.003

Botello-Ramirez, E.; Beltrán-González, B.; Cárdenas-Domínguez, T. (2021). Clima organizacional en los procesos de mejora de la calidad universitaria. Edumecentro, 13(1), 283-289.

Calizaya-López, J.; Bellido-Medina, R. (2020). Clima organizacional y rendimiento laboral en servidores administrativos de una universidad pública de la ciudad de Arequipa. Revista de Psicología, 9(3), 37-55. DOI: https://doi.org/10.36901/psicologia.v9i3.701

Castillo-Martín, L. (2013). Diagnóstico situacional en el Clima organizacional, en la Administración Central del Departamento de Boyacá (Tesis de grado). Universidad Nacional Abierta y a Distancia. Tunja, Colombia. Recuperado de https://bit.ly/3BfPx9m

Charry-Condor, H. (2018). La gestión de la comunicación interna y el clima organizacional en el sector público.Comuni@cción,9(1),25-34.

Chiang-Vega, M.; Salazar-Botello, M.; Huerta-Rivera, C.; Núñez-Partido, A. (2008). Clima organizacional y satisfacción laboral en organizaciones del sector estatal (Instituciones públicas). Desarrollo, adaptación y validación de instrumentos. Universum, 23(2), 66-85. DOI: https://doi.org/10.4067/S0718-23762008000200004 
Del Toro-Granados, J.; Salazar-Sotter, M.; Gómez-Rangel, J. (2011). Clima organizacional, satisfacción laboral y su relación con el desempeño laboral en trabajadores de una PYME de servicios de ingeniería. Clío América, 5(10), 204-227. Recuperado de https://bit.ly/3EqmGkr

Escurra-Mayaute, L. (1988). Cuantificación de la validez de contenido por criterio de jueces. Revista de Psicología, 6(1-2), 103-111. Recuperado de https://bit.ly/3EhKNSq

Fernández-Oliva, D.; Revilla-Velásquez, M.; Kolevic-Roca, L.; Cabrejos-Castilla, I.; MuchaypiñaGallegos, I.; Sayas-Aviles, I.; Chávez-Conde, L.; Mamani-Urrutia, V. (2019). Clima organizacional y satisfacción del usuario externo en los servicios de hospitalización del Instituto Nacional de Salud del Niño, 2017. Anales de la Facultad de Medicina, 80(2), 188-192. DOI: https://doi.org/10.15381/anales.802.15745

García-Velázquez, R.; Hernández-García, T.; Navarrete-Zorrilla, D.; Corichi-García, A.; SánchezMonjaraz, G. (2014). Elementos determinantes del clima organizacional. European Scientific Journal, 10(28), 107-122. Recuperado de https://bit.ly/3bfCtGn

Gómez, C. (2001). Diseño, construcción y validación de tres instrumentos para medir el clima organizacional en instituciones de educación superior desde la teoría de la respuesta al ítem. Cuadernos Hispanoamericanos de Psicología, 1(2), 123-154.

Gómez-Rada, C. (2004). Diseño, construcción y validación de un instrumento que evalúa clima organizacional en empresas colombianas, desde la teoría de respuesta al ítem. Acta Colombiana de Psicología, (11), 97-113.

Gonzáles-Vásquez, J.; Ramírez-López, R.; Terán-Ayay, N. T.; Palomino-Alvarado, G. del P. (2021). Clima organizacional en el sector público Latinoamericano. Ciencia Latina Revista Científica Multidisciplinar, 5(1), 1157-1170. DOI: https://doi.org/10.37811/cl rcm.v5i1.318

González-Bonilla, Y. (2019). Competencias laborales y clima organizacional como predictores del desempeño organizacional en una institución de educación superior privada. Ciencia Administrativa, (2), 8-16. Recuperado de https://bit.ly/3vUU1RB

González-Millán, O.; Rodríguez-Díaz, M.; Molina-Valencia, R. (2011). Medición de seguridad y clima organizacional en la minería sogamoseña. En XVI Congreso Internacional de Contaduría, Administración e Informática. UNAM, Ciudad de México, México, 5-7 de octubre. Recuperado de https://bit.ly/3jKP7lw

Hair, J.; Anderson, R.; Tatham, R.; Black, W. (2014). Análisis multivariante. México: Prentice Hall.

Hernández, R.; Fernández, C.; Baptista, P. (2014). Metodología de la investigación. (6ª ed.). Ciudad de México: McGraw-Hill.

Hernández-Sampieri, R.; Méndez-Valencia, S. (2012). Exploración factorial del clima y la cultura organizacional en el marco del modelo de los valores en competencia. Psique Mag, 1(1), 1-37.

Hultell, D.; Gustavsson, J. (2008). A psychometric evaluation of the satisfaction with life scale in a Swedish nationwide sample of university students. Personality and Individual Differences, 44(5), 1070-1079. DOI: https://doi.org/10.1016/j.paid.2007.10.030 
Iglesias-Armenteros, A.; Sánchez-García, Z. (2015). Generalidades del clima organizacional. MediSur, $13(3), 455-457$.

Johnson, B.; Stevens, J. J. (2001). Exploratory and confirmatory factor analysis of the School Level Environment Questionnaire (SLEQ). Learning Environments Research, 4(3), 325-344. DOI: https://doi.org/10.1023/A:1014486821714

Katz, M. (2006). Multivariable analysis (2a ed.). Cambridge: Cambridge University Press. DOI: https://doi.org/10.1017/CB09780511811692

Kline, R. (2011). Principles and practice of structural equation modeling (3a ed.). Nueva York: Guilford.

Lara-Hormigo, A. (01 de enero de 2014). Introducción a las ecuaciones estructurales en Amos y $R$. Universidad del Valle de México (UVM) - Chihuahua. Recuperado de https://bit.ly/2ZrjwxZ

Likert, R. (1967). The human organization. Nueva York: McGraw-Hill.

Marsh, H.; Lüdtke, O.; Nagengast, B.; Morin, A.; Von Davier, M. (2013). Why item parcels are almost - never appropriate: Two wrongs do not make a right--camouflaging misspecification with item parcels in CFA models. Psychological Methods, 18(3), 257-284. DOI: https://doi.org/10.1037/a0032773

Merino-Soto, C.; Livia-Segovia, J. (2009). Intervalos de confianza asimétricos para el índice la validez de contenido: un programa Visual Basic para la V de Aiken. Anales de Psicología, 25(1), 169171. Recuperado de https://bit.ly/3nuvLlA

Meza-Barajas, E. (2017). Análisis en la percepción del género entre clima organizacional y satisfacción laboral del sector industrial. Comuni@cción, 8(2), 148-158.

Ministerio de Salud del Perú. (2011). Documento técnico: metodología para el estudio del clima organizacional. Lima: Comité Técnico de Clima Organizacional. Recuperado de https://bit.ly/3miZpe1

Montoya-Cáceres, P.; Beiio-Escamlila, N.; Bermúdez-Jara, N.; Burgos-Ríos, F.; Fuentealba-Sandoval, M.; Padilla-Pérez, A. (2017). Satisfacción laboral y su relación con el clima organizacional en funcionarios de una universidad estatal chilena. Ciencia \& Trabajo, 19(58), 7-13. DOI: https://doi.org/10.4067/S0718-24492017000100007

Palafox-Soto, M.; Ochoa-Jiménez, S.; Jacobo-Hernández, C. (2019). La cultura organizacional como base para la permanencia en las organizaciones. Revista San Gregorio, (35), 198-207. DOI: https://doi.org/10.36097/rsan.v1i35.1109

Pecino-Medina, V.; Mañas-Rodríguez, M.; Díaz-Fúñez, P.; López-Puga, J.; Llopis-Marín, J. (2015). Clima y satisfacción laboral en el contexto universitario. Anales de Psicología, 31(2), 658-666. DOI: https://doi.org/10.6018/analesps.31.2.171721

Revilla, R.; Acosta, C.; Reyna, C. (2013). Estudios de validez y confiabilidad de la escala de compra impulsiva en jóvenes y adultos de la ciudad de córdoba, Argentina. Ciencias Psicológicas, 7(2), 133-142. DOI: https://doi.org/10.22235/cp.v7i1.1054 
Riveros-Paredes, P.; Grimaldo-Muchotrigo, M. (2017). Valores y clima organizacional en docentes de un instituto de educación superior de Lima. Ciencias Psicológicas, 11(2), 179-188. DOI: https://doi.org/10.22235/cp.v11i2.1489

Ruiz, M.; Pardo, A.; San Martín, R. (2010). Modelos de ecuaciones estructurales. Papeles del Psicólogo, 31(1), 34-45.

Schumacker, R. E.; Lomax, R. G. (2004). Una guía para principiantes sobre el modelado de ecuaciones estructurales (2 ${ }^{\underline{a}}$ ed.). Londres: Lawrence Erlbaum Associates.

Sotelo-Asef, J.; Figueroa-González, E. (2017). El clima organizacional y su correlación con la calidad en el servicio en una institución de educación de nivel medio superior. RIDE. Revista Iberoamericana para la Investigación y el Desarrollo Educativo 8(15), 582-609. DOI: https://doi.org/10.23913/ride.v8i15.312

Tapias-Cuadrado, A. (2014). Diagnóstico del clima organizacional en el área administrativa de la Universidad Tecnológica del Chocó (Diego Luis Córdoba) (Tesis de maestría). Universidad Nacional de Colombia. Bogotá, Colombia. Recuperado de https://bit.ly/3pFLlO5

Toro-Álvarez, F. (2001). El clima organizacional: perfil de empresas colombianas. Medellín: Cincel.

Ventura, J.; Caycho, T. (2017). El coeficiente Omega: un método alternativo para la metodología de confiabilidad. Revista Latinoamericana de Ciencias Sociales, Niñez y Juventud, 15(1), 625-627.

Williams, L. (2013). Estudio diagnóstico de clima laboral en una dependencia pública (Tesis de maestría). Universidad Autónoma de Nueva León. Monterrey, México. Recuperado de https://bit.ly/3bf8zlt 